\title{
Evaluation of Spatial Distribution of Secondary Schools in Hebron City / Southern West Bank
}

\author{
Hassan Qaddumi \\ Department of Applied Geography, Hebron University, Hebron, Palestine
}

Email address:

hassanq@hebron.edu

\section{To cite this article:}

Hassan Qaddumi. Evaluation of Spatial Distribution of Secondary Schools in Hebron City / Southern West Bank. Advances in Networks. Vol. 8, No. 1, 2020, pp. 9-15. doi: 10.11648/j.net.20200801.12

Received: September 28, 2019; Accepted: February 21, 2020; Published: October 30, 2020

\begin{abstract}
This research dealt with the study and evaluation of the reality of secondary schools in the city of Hebron, and compared with the planning standards, and this is one of the important foundations through which to draw the current picture of the reality of these schools, to form a basis on which to support the current and future planning proposals necessary for the development of the reality of those Therefore, this research relied on the quantitative analytical approach as the most appropriate method for expressing statistical analyzes, as well as using it in conducting some of the analyzes available in the GIS program. Therefore, the research has come up with a set of results, including the results of statistical analysis that indicate that the characteristics of the majority of the secondary teacher was incompatible with their planning standards, due to the prevalence of random pattern in the signing of these schools, as well as not to rely on the foundations and planning criteria for the distribution of uses shown And pitches in them.
\end{abstract}

Keywords: Planning Standards, Spatial Fit, Site Selection, Capacity, Accessibility

\section{Introduction}

The degree of excellence of any science stems from the amount of its utility to serve the community, and the fact that geography is aware of the importance of spatial studies in terms of highlighting the geographical location and features, limits and characteristics of natural and human, and other studies that contribute to the development of society, and services are different types of the most important components of communities Including the educational services, which is one of the most important community services and that are of interest and study by the geographers because of the great impact on the lives of the population as they represent the basis on which the city in the development of civilization and social role through the development of the atmosphere Scientific and cultural community, where Knapp schools at different levels represent a scientific base that enables people to gain knowledge about various issues [6].

The study and evaluation of the spatial distribution of secondary schools and the adequate knowledge of their different characteristics is one of the important studies because of their association with the factors of the population and the place and the associated developments and changes. In order to maximize the benefit of the population and in proportion to the characteristics of the place [2], This study is an attempt to clarify the geographical contribution to the analysis and evaluation of the reality of secondary schools in the city of Hebron, as well as to make some suggestions to the competent authorities in this vital sector in the belief that the role of Geographically played in the development process.

\section{The Study Area}

The city of Hebron is one of the oldest cities in the world and dates back more than 6000 years. It was built in a location that has special characteristics that contributed to its growth and development. It is located in the south west of the West Bank on a long line 35:8 east and latitude 31:31 north. $25 \mathrm{~km}^{2}$ ). According to the Palestinian Central Bureau of Statistics (PCBS) for 2015, the population was approximately 208,750 [11]. 


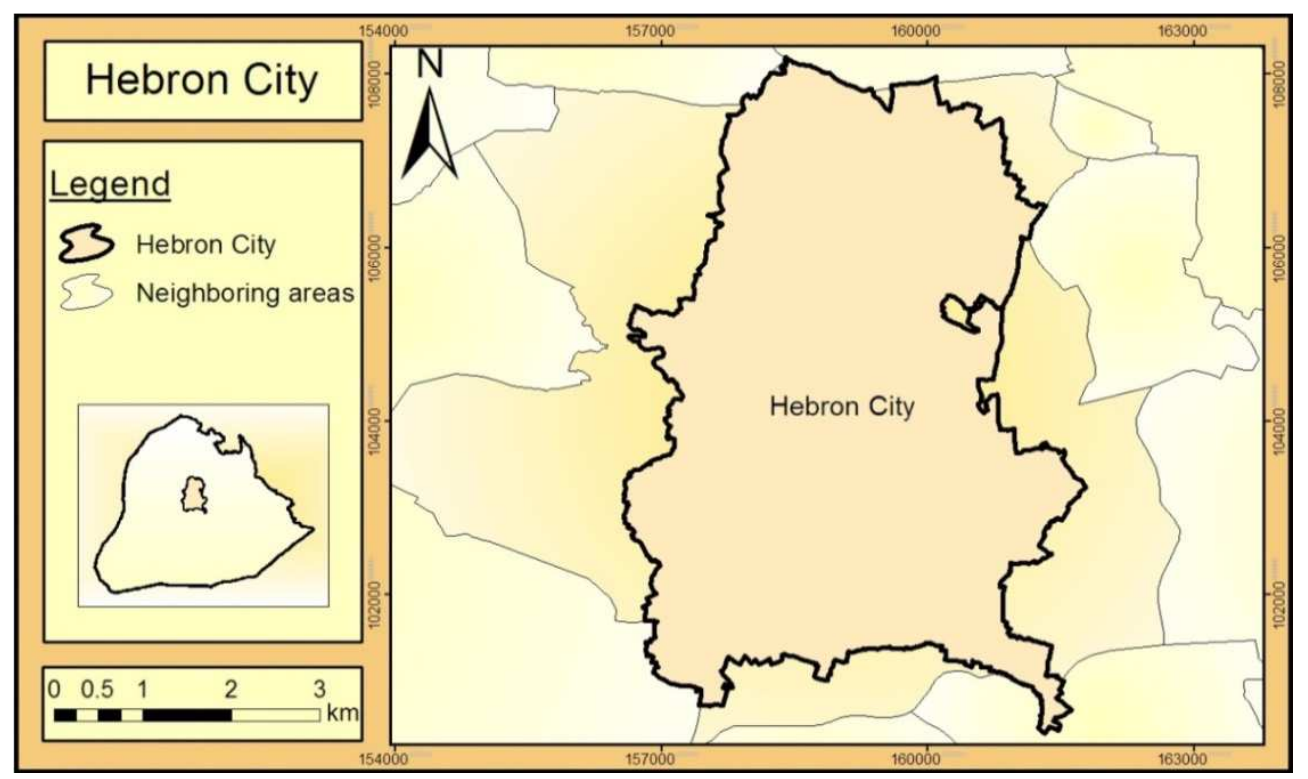

Figure 1. The study area.

\section{Study Problem}

As in other Palestinian cities, Hebron suffers from randomization in the spatial signature of secondary schools. This has resulted in the absence of spatial justice in distribution and the negative effects such as the difficulty of reaching some of these schools. Achieving a level of efficiency and efficiency in it since the growth of this service is associated with population growth and urban population increase demand is growing and is supposed to grow commensurate with the urban growth, thus the problem of the study in an attempt to assess and monitor the reality Secondary schools in the city of Hebron and to measure the compatibility and harmony between the characteristics of these schools and their planning standards.

\section{The Importance of Study}

The importance of this study is in two aspects:

1. The scientific aspect: is the vitality of the idea of the study and based on trying to shed light on one of the important topics that contribute to the service of the population thus play an important role in the development of society.

2. The practical aspect: It is reflected in how to use the technique gis to represent the current image of these schools and the output of the future image according to the foundations and planning standards of their own to contribute to clarify the vision of decision makers and solutions to the problems facing these schools.

\section{Objectives}

This study sought to achieve the following objectives:

1. Measurement and analysis of the characteristics of secondary schools in quantitative and spatial terms.
2. Evaluate the efficiency and adequacy of these schools by comparing them with international planning standards.

3. To propose realistic plans with geographical visions to solve the problems experienced by secondary schools.

4. Work on assessing the current and future needs of secondary schools.

5. Work on the production of figures showing the best sites for the establishment of secondary schools in the study area, in accordance with their planning standards.

\section{Study Questions}

1. To what extent do secondary school characteristics in the study area match the planning criteria?

2. How many students are assigned to each teacher in secondary schools in the study area?

3. How much is the absorptive capacity of each school, and what is the size of the surplus or deficit?

4. How easy are students to access their schools, and what are the factors that hinder it?

\section{The Study Methodology}

The quantitative analysis method is used as the most appropriate method for expressing statistical analysis, as well as using it to perform some analysis available in the gis program.

\section{The Spatial Distribution of Secondary Schools in Hebron}

Secondary schools are important services in any society because they constitute a solid foundation that contributes to the formation of a conscious generation that contributes to the service of their homeland. The spatial analysis begins by 
explaining the spatial distribution of these schools [10].

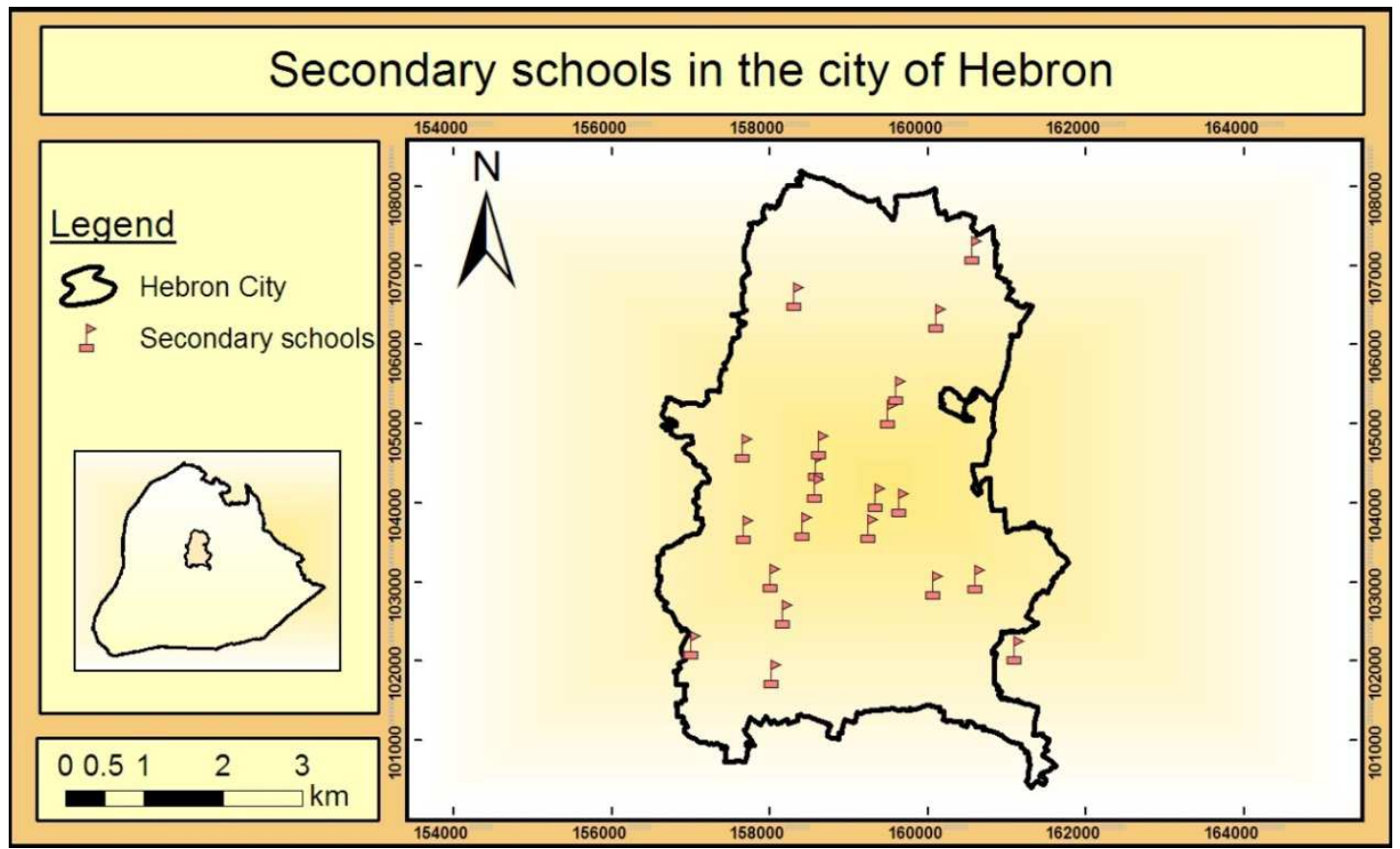

Figure 2. High schools in Hebron.

Figure 2 shows the secondary schools in the city of Hebron with 21 schools, including one mixed school, 7 male schools and 13 girls' schools [8].

\subsection{Spatial Analysis of the Reality of High Schools and Their Conformity with Spatial Planning Criteria}

The process of analyzing and evaluating the reality of secondary schools is carried out by identifying their spatial characteristics and determining their conformity with the planning criteria, in terms of: the location of the school for land use, the urban growth trends as shown in the following figure 3 , as well as the accessibility assessment. The process of spatial assessment of the reality of these schools must include a degree of safety and privacy, because it is important to clarify the problems experienced by these schools [3]. Accordingly, the following table 1 illustrates the global planning standards for secondary school locations.

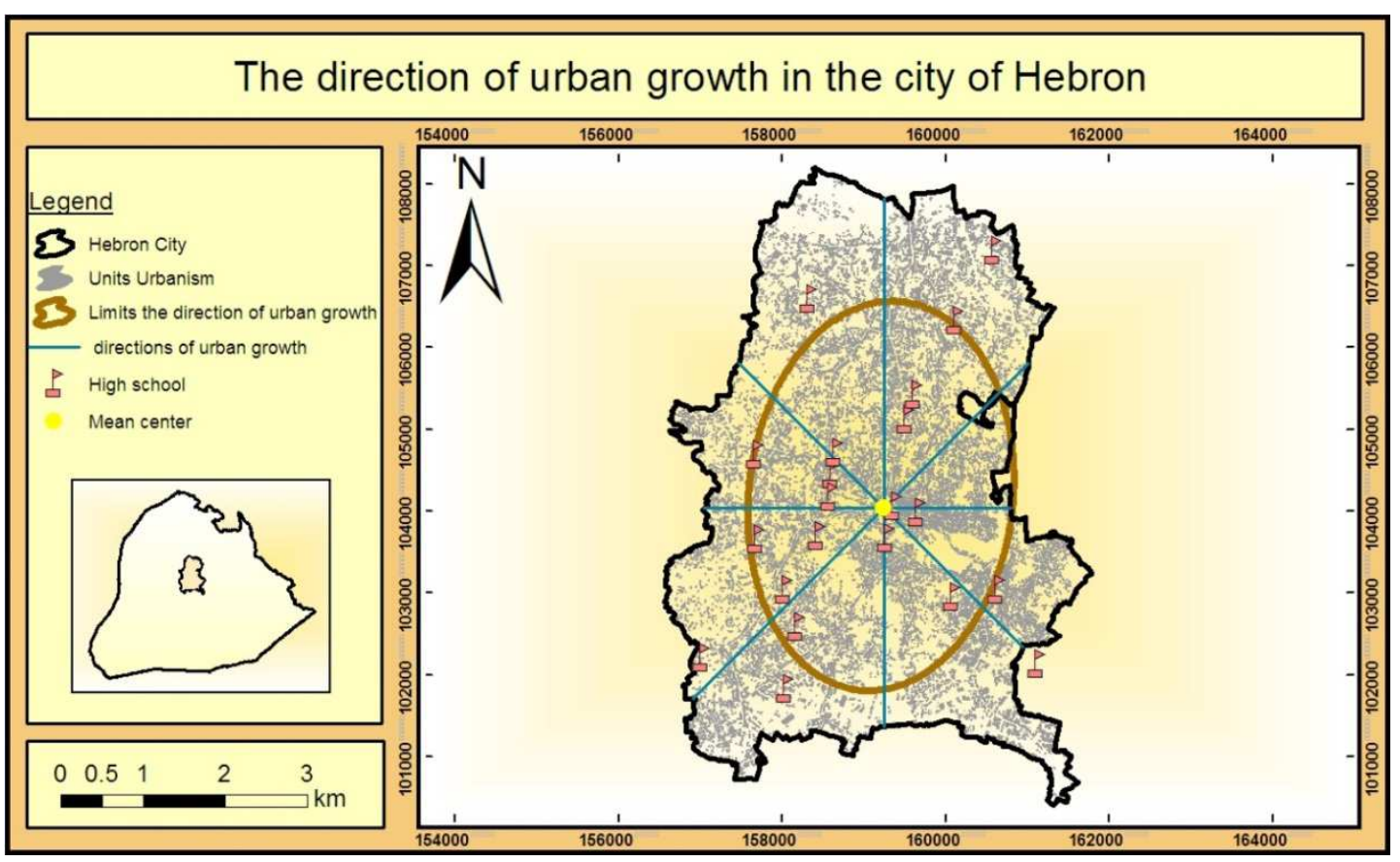

Figure 3. Shows the direction of urban growth in the study area. 
Table 1. Global planning standards for secondary school locations.

\begin{tabular}{|c|c|c|}
\hline \multicolumn{2}{|l|}{ Standard } & Items \\
\hline Admission & For land uses: & The location is in a residential neighborhood and not a commercial or industrial neighborhood. \\
\hline General: & For the direction of urban growth: & The site should be in the direction of the expected growth of the city. \\
\hline & Depending on the type of street: & To be located on the sub street. \\
\hline Accessibility: & Due to congestion: & Easy access and exit from the site without exposure to car congestion. \\
\hline & Depending on availability of transportation: & The possibility of easy transportation to and from traffic. \\
\hline Topographic & For heights: & Choose relatively high positions to increase exposure to wind. \\
\hline Location: & For mileage: & Site tendencies are not more than $2 \%$. \\
\hline Environmental & For safety degree: & To be far from the target security areas we accept the Israeli occupation. \\
\hline standards: & For privacy: & The location of the school must be in a quiet environment, away from the causes of pollution. \\
\hline
\end{tabular}

The previous figure shows the trend of urban growth in the study area. The oval shape shows that the urban growth trend of the city is in the north direction, since it is the longest axis of the growth directions axis starting from the area of the corner gate as the center of the city and to the city limits to the north, With a length of $(3.78 \mathrm{~km})$ [9].
Based on the planning criteria in Table 1 of the Global Planning Standards for Secondary Schools Sites, the characteristics of sites in which secondary schools are located in the city of Hebron have been analyzed. This is illustrated in Table 2 of the spatial analysis of the characteristics of secondary schools In the city of Hebron.

Table 2. Spatial Analysis of Characteristics of Secondary Schools in Hebron City.

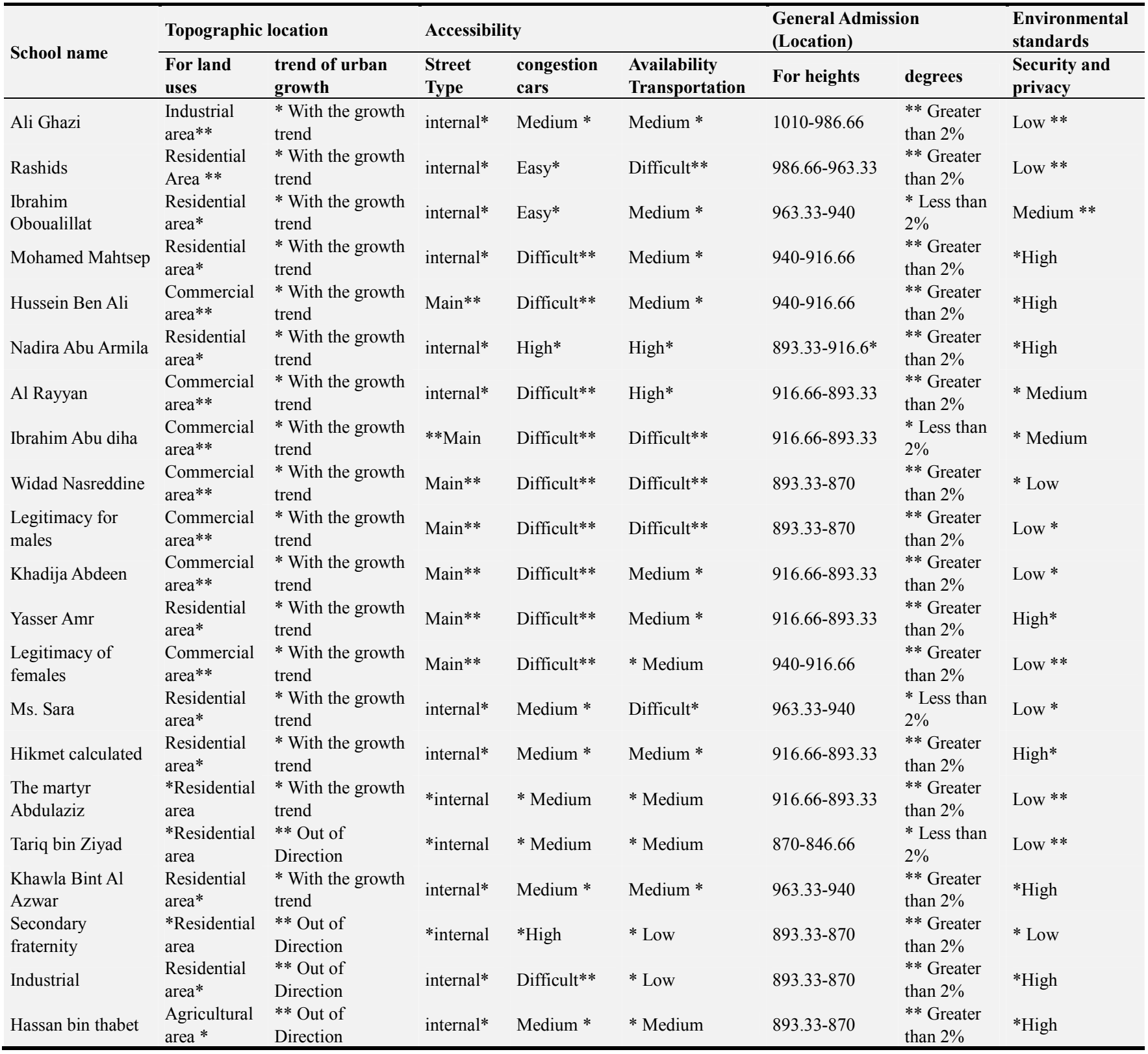


Table 2 presents the results of the spatial analysis of the secondary school characteristics in the study area in comparison with the planning criteria. These schools were divided according to their suitability to the planning criteria to (3) levels, as follows:

First, the first level reflects the schools whose location characteristics corresponded to the planning criteria, such as: Ibraheem Abu al-Daba'at Secondary School for Girls whose characteristics were as follows: With regard to the general acceptance of the type of land use and the direction of urban growth, In the direction of urban growth of the city, [8] and when analyzing the characteristics of this school, we find it located in a residential neighborhood known as Wadi Abu Aktayle, which corresponds to the trend of urban growth in the study area, which takes the northern direction, as shown in the previous figure 3 VIA appropriate characteristics with planning standards set came in Table 1 Previous.

Which stipulate that high schools should be located in high places and no more than (2\%). As for the characteristics of this school, it is located in an area between $940-963.33 \mathrm{~m}$, while its slope is between $0-0.24 \%$. It is located on $\mathrm{Abu}$ Ektila Street, The planning criterion, which indicates the need for secondary schools on the internal streets, where it is easy to access due to the lack of traffic congestion in that area, and the average access depending on the factor of the availability of transport, and for environmental standards that reflect the degree of safety and privacy, which emphasizes the need to be The location of the school is far from any kind of hazards such as the power supply line and Masad Pollution and be away from the security areas and targeted by the Israeli occupation, as well as to achieve the privacy of the school, we find the middle case because of the neighboring sources of pollution as the presence of more than a specific near it, in contrast there is a degree of privacy that is a school for females being surrounded by a fence and an external gate [1].

The school is also located in the neighborhood of Qarn alThawr, which is known as a residential neighborhood. It is compatible with the urban growth trend in the city. The street on which the school is located is an inner street known as the Horn of the Bull. This measure measures the extent of accessibility according to road congestion and when applying this criterion to the reality of this school we can say that its geographical position has contributed to the absence of the impact of car congestion to find that this criterion does not constitute an effective factor in access therefore does not appear $\mathrm{T}$ his role in delaying the students from the school, as well as the case for the index provides easy transportation access are regarded as being linked to the internal road network in the study area, the topography is found located at an altitude ranging between (893.33-916.66 m), This reflects the compatibility with the schematic requirement in Table 1, which emphasizes the necessity of choosing relatively high locations to increase the exposure to wind in order to provide ventilation for the school. The safety and privacy levels are considered high due to the lack of pollution sources
Therefore, the location of this school has been in violation of the planning criteria described above. NH ranges between (4.95-6.80\%).

The Hikmat Secondary School for Boys is one of the schools covered by this level because it violates one of the planning criteria, which is the degree of tendency to be located in an area of between $6.80-9.01 \%$. In contrast, it matches the planning standards in its other characteristics, Mountain Abu Rumman in the sense that it is located in the residential district is within the limits of the direction of growth and expansion of the city and at a height ranging between (893.33 - $916.66 \mathrm{~m})$, a location devoid of pollution sources to express the high degree of safety and privacy, as well as on an internal street, A life to show us that the possibility of movement of This is medium depending on the availability of traffic and congestion.

The last school included in this classification is Hassan bin Thabit secondary school for girls, located in the second neighborhood of Hawooz in an area classified as agricultural, meaning that it is located on an internal street called Nnqr Street and is known as the average traffic congestion because of taking some of the moving cars between the cities of Dura and Hebron, This means that the access to this school is medium, and that it is at an altitude of $870-893.33 \mathrm{~m}$. It conforms to its planning criterion to allow the solar radiation to enter the school and to provide good ventilation for the school in order to preserve public health, I have $h$ The environmental standards of this school are based on a high safety and privacy index, due to a number of reasons: low traffic congestion on the street leading to it, indicating a decrease in the exposure of students to traffic accidents, and the school has two exit points that can be evacuated quickly in emergencies, And the absence of pollution from the site has a great impact.

It should be noted that the majority of schools classified as Tier 1 are located in areas with a tendency greater than $2 \%$. This is not consistent with planning standards but is considered to be lower than other schools. It was raised through the use of some methods and means during the settlement process because it did not increase significantly.

The second level includes 5 schools with medium characteristics corresponding to the planning criteria, such as: Mohammed Ali Al-Muhtasib School located in the neighborhood of Ein Sara-Sharq. It is compatible with the urban growth trend as shown in the previous figure 3 (916.66 - $940 \mathrm{~m}$ ), and according to the standards of environmental standards, it is classified as high safety because the site is free from sources of pollution, and the possibility of rapid evacuation of the school in case of emergency came in support of this trend, despite these characteristics Characteristic of this school but its other characteristics were a The difficulty of reaching students because of the congestion of vehicles on the road, despite the existence of a private entrance to them, but the reason for the congestion here is the passage of students from the street Ain Sarah, which is a vibrant arteries in the city of Hebron, where the 
movement of active population lead to form The rush hour (morning - noon) is the period during which students move to and from school. Due to the influence of the previous factor, accessibility in terms of availability of vehicles has been achieved at the intermediate level, and other criteria that came in support of the classification of this school within this level is It occurred in the area of Witter And $\mathrm{h}$ decline between (9.01-11.56\%) in the sense that it violates your schematic criterion in this direction

Al-Rayyan Secondary School for Girls is ranked second in this level. Table 2 shows that its spatial characteristics coincided with the planning criteria in several aspects, such as: (833.33 $-916.66 \mathrm{~m})$, which was classified as being in violation of the Regression Regression Standard because it lies in an area with a slope between (6.81-9.01\%), This is due to the active movement of the vehicles in this vital area that connects between the skin and the center of the city of Hebron. Nevertheless, it has violated the normative basis of planning, as it is located in the neighborhood known as a commercial neighborhood The active movement of the population and their goods, which reflected negatively on access according to the factor of traffic congestion on the roads because the trucks loaded with goods taken from this street a course, which in turn led to the presence of noise pollution affecting the process of the educational process, Canet school evacuation in emergency situations are considered medium and this is what has been inferred through this special school questionnaire model

Thirdly, Mrs. Sara Secondary School for Girls was one of the teachers at this level. It was located between 940-963.33 $\mathrm{m}$ on the internal street of Al-Mansur in Al-Manshar neighborhood. This was in line with the trend of the city's growth and its other characteristics which met the criteria The criteria for accessibility have had a greater role in the presence of this school within this level due to the difficulty of the availability of transportation, and the possibility of evacuation of the school in emergency situations is moderate due to the presence of an entrance And only one director [7].

In addition to the above mentioned schools, both the Abdul Aziz Abu Asnayah Secondary School for Girls and the Khawla Bint Al Azur Secondary School for Girls show that there are some characteristics that are compatible with the planning standards of both schools because they are located in residential neighborhoods where the school The first is located in the Abu Asnayah and the other in Jabal Abu Rumman. The common denominator between them is the accessibility, which takes a medium degree according to the standards of traffic congestion and availability [2], and when compared with environmental standards, the Martyr Abdul Aziz Abu Asnina School is surrounded by sources Pollution as used car structures, therefore, the degree of safety and privacy is low, unlike the school Khawla bint Al Azur, whose environment is free of sources of pollution.

After reviewing the schools with the corresponding characteristics and the medium corresponding to the planning criteria, it was necessary to clarify the teacher, which was characterized as incompatible with these standards [6] and the negative effects such as the presence of pollution, for example, it can be said that the majority of schools classified in this classification have similar characteristics We find them located in commercial districts that have a negative reflective in accessibility.

In the beginning, we will review the characteristics of Wadad Nasser Al-Din Secondary School for Girls, Khadija Abdeen Secondary School for Girls, Husein Bin Ali Secondary School and Ibrahim Abu Diya Secondary School for Boys due to their presence in commercial districts: Wadi Al-Tuffah neighborhood, Al-Shalala neighborhood, Ein Sara neighborhood, The above mentioned schools are located on the main roads, which in turn reflects the difficulty of access due to the impact of traffic congestion and traffic congestion. It is not meant that there are no cars leading to it. In addition, the Wadad Nasiruddin School suffers from a sewerage network behind the school. The above-mentioned schools suffer from noise pollution resulting from the movement of residents, cars and sellers.

This is not the case in my secondary secondary school for males and the secondary legitimacy of females because they are located in commercial districts and on a main road which is considered the artery of life in the city of Hebron. This is the street of peace, which is witnessing an active traffic movement that expresses a traffic crisis affecting access and contributing delay. Students from their schools, since they are private schools for students from different areas of Hebron governorate and require more than one means of transport to reach them, often use the internal transportation which is experiencing the busiest peak period, and the presence of sources of pollution near them led to the lack of The school's legal school for males suffers from the presence of air pollution caused by the smoke from the bakery of Khalil Rahman, which is next to it, while the legitimate school for girls from the presence of disturbance by the specific near them, and also participate in noise pollution caused by traffic congestion on the Roads [5].

This is contrary to the planning standards that emphasize the need for the school to be located in a residential neighborhood to facilitate the provision of educational service to students, and because of its location is affected by the movement of vehicles belonging to These factories contribute to the formation of noise and air pollution as well as lead to the existence of traffic crisis sometimes, and the passage from the area of Ras al-Jura to access this school contributes to make it moderate in terms of accessibility.

The characteristics of the Al-Rashidi Secondary Boys School in Table 2 above made it one of the schools included in this level because it is located in an area that ranges between $4.59-6.80 \%$. An internal road that does not witness an active population movement has a negative impact on accessibility, and the possibility of evacuation in emergency situations is moderate due to the presence of one entrance and exit.

This level also includes Yasser Amr Secondary School for Girls, Tariq Bin Ziyad Secondary School for Boys and Hebron Industrial Secondary School. When we address the 
characteristics of these schools, we find that they do not conform to the planning standards. Yasir Amr School is located in Jabal Al Rahma on Main Street Known as the street of mercy and this makes them difficult to access due to traffic congestion.

he Tariq Bin Ziyad school in the Israeli-controlled Jabal Jowhar is located in the $\mathrm{H} 2$ area and is therefore outside the boundaries of the growth and spatial expansion of the city of Hebron. This affects access due to the passage of some students to many checkpoints to reach the school Or their behavior to another road, which leads to delays sometimes, and this is what was derived from the survey of the views of students from the questionnaire on this trend, and environmental standards, we find that the degree of safety and privacy is high surrounded by fences and gates of iron surrounds them from the inside and outside may have been the political side Has greatly affected the upgrading of these equipment to protect its teaching staff and students from Israeli harassment [4].

As for the Hebron Industrial High School, it is located in Wadi Al-Hariya, meaning that it is outside the boundaries of the city's urban development. Due to the congestion of vehicles on Al-Hawooz Street, which is one of the streets leading to this school, it takes time to reach it. Who are interested in professional work from different parts of the Hebron governorate to find them passing through other major roads where traffic is crowded and this is another factor that influences the students' access to their school.

\subsection{The Results}

Through the study and evaluation of the reality of secondary schools in the city of Hebron, a number of results were reached:

1. Based on the results of spatial analysis of the reality of secondary schools in the study area, it was found that some schools have met their spatial characteristics in accordance with the planning criteria, including: Ibrahim Abu Al Dababat Secondary School, Al Hajja Nadira Abu Ermilah School, Hekmat Al Muhtaseb School and Hassan Bin Thabit Secondary School for Girls.

2. The results of the statistical analysis indicate that the characteristics of the majority of the secondary teacher in the study area were inconsistent with their planning standards, due to the prevalence of random pattern in the signature of these schools

3. As well as not to rely on the foundations and planning standards for the distribution of uses shown and playgrounds in those schools.

4. The majority of secondary schools suffer from inefficiency in terms of capacity.
5. Where the surplus rate in the number of students about (1706 students), including (1138 students) and (568 students). This indicates the need to add two schools for girls and one secondary school for males.

\section{Recommendations}

1. Relying on geographical information systems for reorganizing and rehabilitating educational centers

2. Emphasis must be placed on the spatial relationships between communities and schools.

3. Attention must be paid to the Palestinian statistics data, so that there will be coordination when distributing schools.

4. The municipality must provide full services to educational schools.

\section{References}

[1] Ismail, Samar: Strategies for Achieving Sustainability in Schools Urban Design - Case Study: UNRWA Schools in Gaza Strip, Master Thesis, Islamic University, 2011.

[2] Palestinian Central Bureau of Statistics, Hebron, 2016.

[3] Jaber, Zeina, Saad, Ali: Spatial Analysis of the Locations of Public Schools in Najaf City Using GIS, Najaf Water Directorate.

[4] Said, Ali, Shaalan, Iman: Evaluation of the Efficiency of Educational and Health Services in the City of Al-Haidariya, Scientific Research Published, Journal of Geographical Researches, No. 19, 2013.

[5] Shuqair, Heba, Distribution and Planning of Educational Services in Salfit Governorate Using GIS Technology, Master Thesis, An-Najah National University, 2009.

[6] Askar, Ahmad: Spatial Analysis of Government Schools in Gaza City Using Geographic Information Systems (GIS) Case Study, Sheikh Ajlin Neighborhood, Master Thesis, Islamic University, 2015.

[7] Atatra, Salam: Preparation of a plan to determine the needs of the Directorate of Education in Qabatiya of school buildings during the next four years, Master Thesis, An-Najah National University, 2008.

[8] Education Directorate - Downtown Hebron, 2016.

[9] Ministry of Municipal and Rural Affairs, Guide to Planning Standards for Saudi Services, 2005.

[10] Yousef, Taher: Spatial Analysis of Educational Services in Nablus City Using GIS Technology, Master Thesis, An-Najah National University, 2007.

[11] http://www.hebron-city.ps/. 